\title{
Photonic crystal couplers for slow light
}

\author{
Andrey A. Sukhorukov ${ }^{a}$, Sangwoo Ha ${ }^{a}$, Kokou B. Dossou ${ }^{b}$, Lindsay C. Botten $^{b}$, \\ Andrei V. Lavrinenko ${ }^{c}$, Dmitry N. Chigrin ${ }^{d}$, and Yuri S. Kivshar ${ }^{a}$ \\ ${ }^{a}$ Nonlinear Physics Centre and Centre for Ultra-high bandwidth Devices for Optical Systems \\ (CUDOS), Research School of Physical Sciences and Engineering, \\ Australian National University, Canberra, ACT 0200, Australia \\ ${ }^{b}$ CUDOS, Department of Mathematical Sciences, University of Technology, \\ Sydney, NSW 2007, Australia \\ ${ }^{c} \mathrm{COM} \bullet \mathrm{DTU}$, Department of Communications, Optics \& Materials, \\ Technical University of Denmark, DK-2800 Kgs Lyngby, Denmark \\ $d$ Physikalisches Institut, Universitat Bonn, Nußallee 12, D-53115 Bonn, Germany
}

\begin{abstract}
We propose a general approach to the design of directional couplers in photonic-crystals operating in the slowlight regime. We predict, based on a general symmetry analysis, that robust switching of slow-light pulses is possible between antisymmetrically coupled photonic crystal waveguides. We demonstrate, through numerical Bloch mode frequency-domain and finite-difference time-domain (FDTD) simulations that, for all pulses with strongly reduced group velocities at the photonic band-gap edge, complete switching occurs at a fixed coupling length of just a few unit cells of the photonic crystal.
\end{abstract}

Keywords: Slow light, photonic crystal, directional coupler

\section{INTRODUCTION}

Waveguides created in planar photonic crystals offer many unique opportunities for manipulating optical pulses that cannot be realized in conventional rib waveguides. In particular, the speed of light can be dramatically reduced, and a series of recent experiments ${ }^{1-5}$ has demonstrated the propagation of slow light associated with the flat regions and extrema in the band-gap dispersion curves. The maximum dynamic tunability of the group velocity of light is possible when the optical frequency is tuned in the vicinity of the photonic bandgap edge. In this regime, however, the effect of frequency dispersion is strongly enhanced and special approaches are required to perform pulse routing, e.g., the modified design of waveguide bends that was introduced for slow light pulses. ${ }^{6}$

In this work, we present a novel and general approach to design directional couplers in photonic crystals where dispersionless tunneling of slow light may be realized. The pulses are fully switched between parallel waveguides at the coupling distance, the value of which remains constant even as the group velocity is varied by some orders of magnitude. The additional advantage of the suggested structure is the short coupling length of only a few unit cells. Such remarkable performance is enabled by the co-existence of modes whose band-edge dispersion is exactly matched, realizing a fundamentally different physical regime in comparison with those considered previously. ${ }^{7-11}$

\section{DISPERSION AND SYMMETRY REQUIREMENTS}

One of the remarkable properties of photonic crystal waveguides is the possibility they afford for reducing the group velocity of optical pulses. The generic mechanism for the slowing of light is associated with the presence of photonic band-gaps. Indeed, the dispersion relation between the optical frequency $(\omega)$ and the Bloch wavenumber $(k)$ in the vicinity of band-edges is commonly expressed as $\omega \simeq \omega_{0}+D_{2}\left(k-k_{0}\right)^{2}$, where $\omega_{0}$ and $k_{0}$ denote the values at the band-edge, and $D_{2}$ is the second-order dispersion coefficient. The group velocity $V_{g}=d \omega / d k \simeq \pm 2\left[D_{2}\left(\omega-\omega_{0}\right)\right]^{1 / 2}$ gradually reduces to zero as the frequency is tuned towards the edge of a transmission band.

Further author information available at the Nonlinear Physics Centre web site: www.rsphysse.anu.edu.au/nonlinear

Advances in Slow and Fast Light, edited by Selim M. Shahriar, Philip R. Hemmer, John R. Lowell,

Proc. of SPIE Vol. 6904, 69040S, (2008) · 0277-786X/08/\$18 · doi: 10.1117/12.772232 


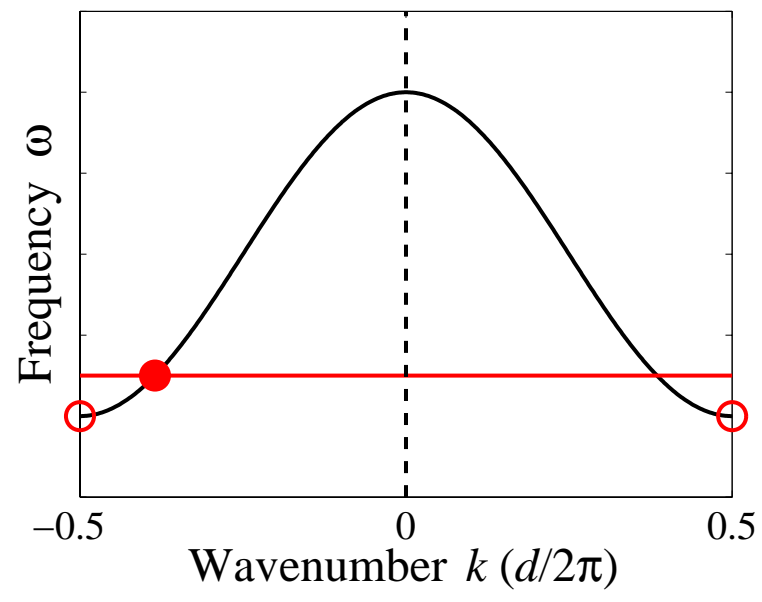

(a)

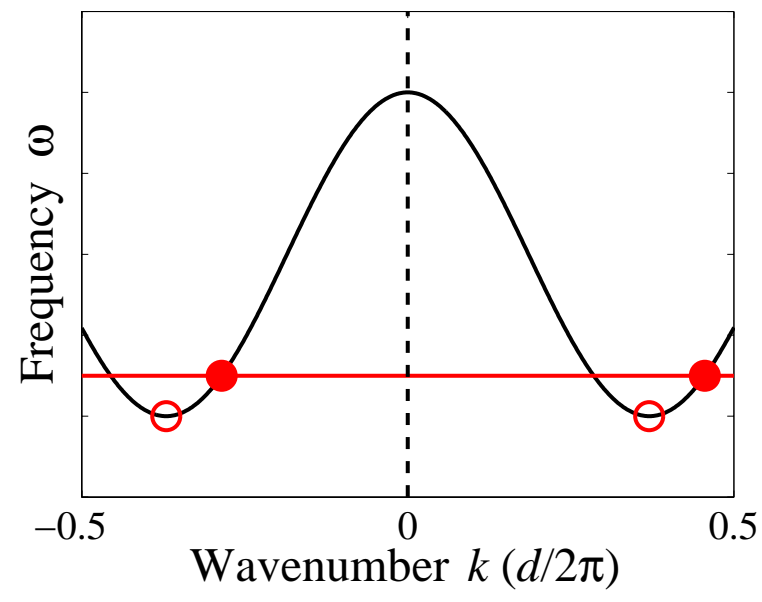

(b)

Figure 1. Characteristic types of band-edge dispersion for slow-light modes in photonic-crystal waveguides. (a) Band-edge is reached at the symmetry points of the Brillouin zone $k=0, \pm \pi / d$. (b) Band-edge is reached inside the Brillouin zone for $k= \pm k_{0} \neq 0, \pm \pi / d$. Open circles mark the lower band-edge, and closed circles - modes with positive slope of dispersion curves (and, accordingly, positive group velocities) for the frequency tuned close to the band-edge.

The concept of directional couplers is based on the effect of periodic tunneling of light between parallel waveguides which are in close proximity to one another. In the case of conventional waveguides, the tunneling can be explained as the beating between co-propagating even and odd super-modes of the coupler. Similar requirements should exist for photonic-crystal couplers, namely that:

(i) there should co-exist two modes which are co-propagating (have the same sign of the group velocity) over a certain range of optical frequencies, and

(ii) these modes should have different symmetry.

Therefore, to realize a directional coupler for slow-light pulses with tunable velocities, it is necessary to satisfy both conditions for a range of frequencies arbitrarily close to the edge of the photonic bandgap.

In order to see how these conditions can be satisfied, we consider the general symmetries of dispersion relations for photonic crystal couplers. The first symmetry is the periodicity of the dispersion relations according to Bloch's theorem,

$$
\omega(k)=\omega(k+2 \pi / d),
$$

where $d$ is the period of the refractive index modulation along the waveguide direction. Moreover,

$$
\psi(x, z ; \omega, k)=\psi(x, z ; \omega, k+2 \pi / d),
$$

where $\psi$ denotes the complex envelope function for the electric or magnetic fields of a Bloch eigenmode. This means that the dispersion points $k, k \pm 2 \pi / d, k \pm 4 \pi / d, \ldots$ are indistinguishable since they correspond to exactly the same distributions of electric and magnetic fields. The second symmetry appears in case of waveguides fabricated in dielectric materials, where

$$
\omega(k) \equiv \omega(-k) \quad \text { and } \quad \psi(x, z ; \omega, k)=\psi^{*}(x, z ; \omega,-k) .
$$

Note that the field profiles for $+k$ and $-k$ are generally different due to the complex conjugation.

According to the second symmetry condition, the band-edges at $\omega=\omega_{0}$ always appear simultaneously at $k=+k_{0}$ and $k=-k_{0}$. However, according to the first symmetry condition, the two modes are identical if 
$k_{0}=0, \pm \pi / d, \ldots$, i.e., when the band-edge appears at the centre or at the edges of the Brillouin zone, as shown schematically in Fig. 1(a). In this case, for a given frequency in the vicinity of the band-gap, there are two intersections with the dispersion curve, where one mode has a positive, and the other a negative, group velocity, such that condition (i) is not satisfied. A completely different situation occurs if the band-edge is inside the Brillouin zone, i.e., $k_{0} \neq 0, \pm \pi / d, \ldots$, as shown in Fig. 1(b). In this case, even as the frequency is tuned arbitrarily close to the band-edge, there appear two pairs of dispersion branches with positive and negative group velocities, and so condition (i) is satisfied.

Having identified the general approach for satisfying the first condition, we now consider condition (ii). The meaning of the latter requirement is that the electric and magnetic fields of the co-propagating modes should be different between the coupled waveguides. Then, the interference of these modes with particular relative phases can produce asymmetric field distributions with maxima in either of the waveguides, thus realizing the operating principle of the directional coupler. Additionally, it is known from the theory of conventional couplers that complete energy transfer between the arms can be achieved when the waveguides are identical, such that the refractive index profile is symmetric, $n(x, z)=n(-x, z)$. In this case, all the eigenmodes are either even, $\psi_{+}(x, z)=\psi_{+}(-x, z)$, or odd, $\psi_{-}(x, z)=-\psi_{-}(-x, z)$, and a pair of such modes would satisfy condition (ii). Indeed, symmetric photonic crystal couplers were shown to perform complete light switching between the arms based on the beating between the odd and even modes. ${ }^{10}$ However, their operation was considered away from the band-edges. In the case of band-edge slow light, we have $\psi\left(x, z ; \omega, k_{0}\right)=\psi^{*}\left(x, z ; \omega,-k_{0}\right)$, and therefore the pair of modes with $k \simeq \pm k_{0}$ are either both even or both odd, such that condition (ii) is never satisfied in symmetric couplers. In contrast, we conclude that photonic crystal couplers can operate for slow-light pulses at the band-edge which appears inside the Brillouin zone [as shown in Fig. 1(b)], with the additional requirement that the dielectric photonic structure is asymmetric with $n(x, z) \neq n(-x, z)$.

A key characteristic of the coupler is the distance over which light tunnels between the arms. In order to calculate the coupling length, we expand the dispersion around the band-edge as

$$
\omega \simeq \omega_{0}+D_{2}\left(|k|-k_{0}\right)^{2}+D_{3}\left(|k|-k_{0}\right)^{3},
$$

where $D_{2}$ and $D_{3}$ are the second- and third-order dispersion coefficients. By inverting these expressions, we obtain the asymptotic dependence of wave-numbers on frequency as

$$
k_{\omega \rightarrow \omega_{0}} \simeq s k_{0}+\sigma\left[\frac{\omega-\omega_{0}}{D_{2}}\right]^{1 / 2}-s \frac{D_{3}}{2\left(D_{2}\right)^{2}}\left(\omega-\omega_{0}\right)
$$

where the values $s= \pm 1$ and $\sigma= \pm 1$ correspond to four different modes. Their group velocities are

$$
V_{g}=\frac{d \omega}{d k} \simeq 2 \sigma D_{2}\left[\frac{\omega-\omega_{0}}{D_{2}}\right]^{1 / 2}+2 s \frac{D_{3}}{D_{2}}\left(\omega-\omega_{0}\right) .
$$

We see that the group velocities of branches with positive $(s=+1)$ and negative $(s=-1)$ wave-numbers asymptotically coincide in the slow-light regime when $\omega \rightarrow \omega_{0}$. The coupling length is defined as the distance over which the phase between the co-propagating modes (both corresponding to $\sigma=+1$ or $\sigma=-1$ ) changes by $\pi$. Since, after each lattice period, the phase of the Bloch modes changes by $(k d)$, we have

$$
L_{c} \simeq \pi d\left|\arg \exp \left\{i\left[k_{s=+1}-k_{s=-1}\right] d\right\}\right|^{-1} \simeq \pi d\left|\arg \exp \left\{i\left[2 k_{0}-D_{3}\left(D_{2}\right)^{-2}\left(\omega-\omega_{0}\right)\right] d\right\}\right|^{-1},
$$

where arg defines the phase of a complex number within the parameter region $[-\pi,+\pi]$. According to this expression, the coupling length approaches a constant value in the slow-light regime as $\omega \rightarrow \omega_{0}$. This remarkable

feature can enable dispersionless tunneling of slow light, where the same tunneling dynamics can be preserved even under the variation of the speed of light by several orders of magnitude.

\section{DESIGN OF SLOW-LIGHT COUPLERS}

We now illustrate how the general requirements formulated in Sec. 2 can be satisfied with a practical coupler design. As an example, we consider two-dimensional photonic crystals created by a hexagonal array of holes in 

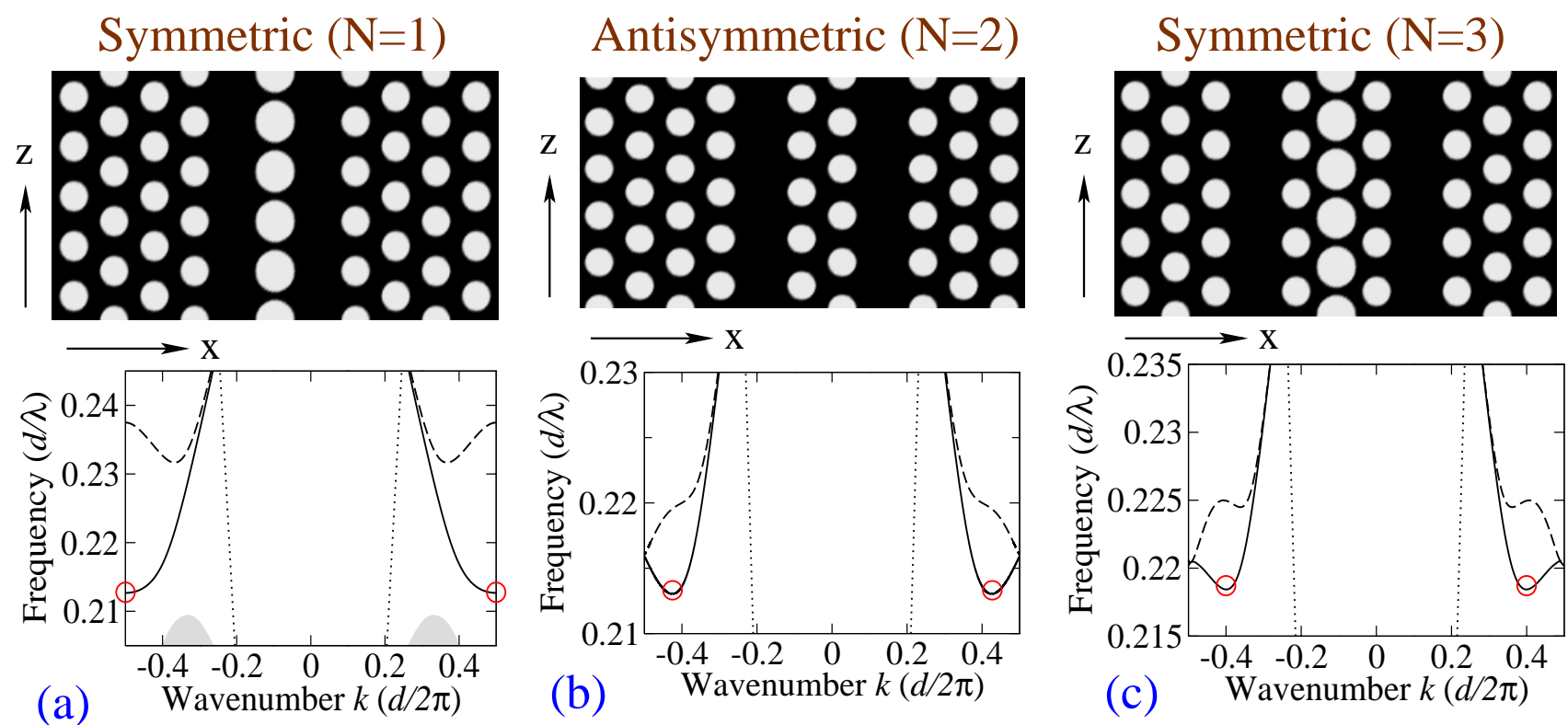

Figure 2. Examples of photonic crystal couplers (top row) and their corresponding dispersion diagram (bottom row) with different number of rows $(N)$ between the W1 waveguides in a hexagonal lattice: (a) $N=1$, (b) $N=2$, and (c) $N=3$. Solid and dashed lines correspond to fundamental and higher-order modes. The hole radius (normalized to the lattice constant) is 0.3 , and 0.42 - for the central row in (a) and (c). Open circles indicate the lower band-edges, where slow-light modes are considered. Dotted lines mark the light-lines, and grey shading in (a) marks the 2D photonic band.

$\mathrm{Si}$ (dielectric constant $\varepsilon=11.68$ ), with a hole radius of 0.3 of the lattice constant. A W1 waveguide is created when a single row of holes is absent. Due to the hexagonal lattice geometry, the coupler symmetry critically depends on the number of rows $(N)$ between the two W1 waveguides. When $N$ is odd, the coupler is symmetric with respect to reflection about a central line between the waveguides $(x \rightarrow-x)$, see the examples for $N=1$ and $N=3$ in Figs. 2(a) and (c). When $N$ is even, then the coupler becomes anti-symmetric, as it maps onto itself only when a reflection is performed simultaneously along two axes $(x \rightarrow-x$ and $z \rightarrow-z)$, see the example for $N=2$ in Fig. 2(b).

We calculate the modal dispersion for the two-dimensional photonic-crystal couplers by computing eigenmodes of Maxwell's equations using the freely available MIT Photonic Bands (MPB) software package. ${ }^{12}$ The dispersion of the TE modes (magnetic field polarized in y-axis) was computed in the frequency range of the two-dimensional photonic band gap. The dispersion curves, corresponding to the coupling between the fundamental modes of individual waveguides, are presented in Fig. 2, bottom row.

According to the general analysis, symmetric couplers, such as those shown in Fig. 2(a) and Fig. 2(c), cannot be used to switch band-edge slow light pulses. It is useful to consider in more detail the reasons for such limitations. First, we analyze the coupler with $N=1$, where we increase the size of the central row of holes (to 0.42 of the lattice constant) in order to move the band-edge above the $2 \mathrm{D}$ photonic band (shown with grey shading in Fig. 2(a), bottom). We see that the coupler with $N=1$ has the lower band-edge at $k= \pm \pi / d$, which means that there exists only one forward-propagating mode in the slow-light regime. Additionally, such a mode should be either symmetric or antisymmetric, corresponding to equal energy distribution between the arms. Indeed, the finite-difference time-domain (FDTD) simulations presented in Fig. 3 show that the pulse coupled to a single waveguide at the input, spreads equally between the two waveguides as it propagates along the structure, and that periodic tunneling between the waveguides does not occur. Note that the input of the second waveguide is closed by three holes to avoid direct coupling to it from the source. Although some degree of pulse splitting is visible in simulations, probably due to complicated excitation dynamics, this does not affect the symmetry between the waveguides.

In order to satisfy the first requirement formulated in Sec. 2 and move the band-edges inside the Brillouin zone, we optimize the design of the $N=3$ coupler by changing the size of the central row of holes (to 0.42 of 


\section{Symmetric coupler $(\mathrm{N}=1)$}
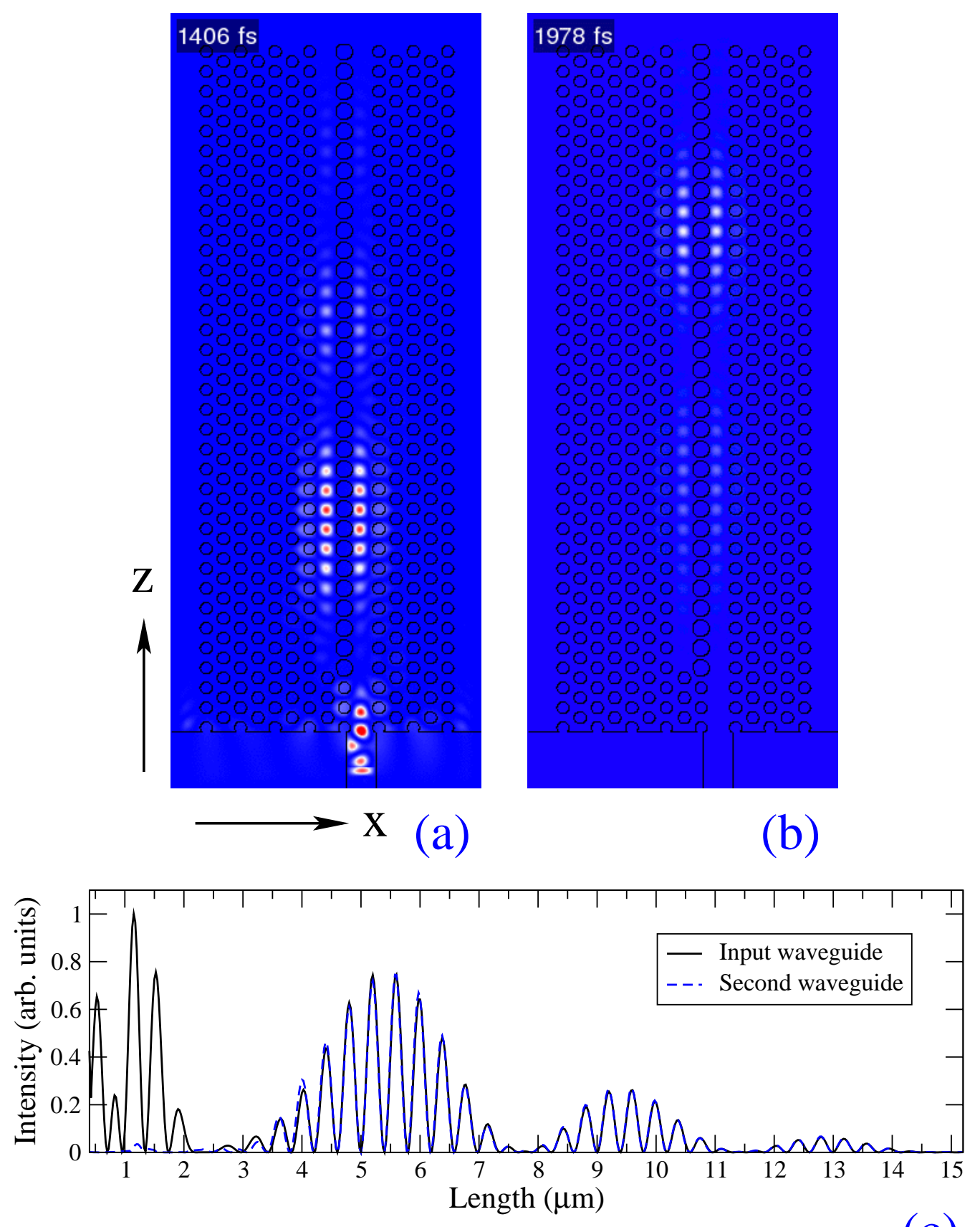

(c)

Figure 3. Simulations of pulse propagation through the symmetric coupler shown in Fig. 2(a), top. The central frequency of the pulse $(\omega=0.21238)$ is tuned close to the gap-edge, indicated by the open circle in $2(\mathrm{a})$, bottom. (a,b) Snapshots of the magnetic field intensity at different time steps, as indicated by labels. (c) Field intensities along the centres of two coupled waveguides, at the time frame corresponding to plot (a). The lattice constant is 400nm, the input pulse duration is $500 \mathrm{fs}$, and the corresponding normalized pulse bandwidth $\Delta \omega=0.002$. The estimated pulse velocity is $V_{g} \simeq c / 115$. 


\section{Symmetric coupler $(\mathrm{N}=3)$}
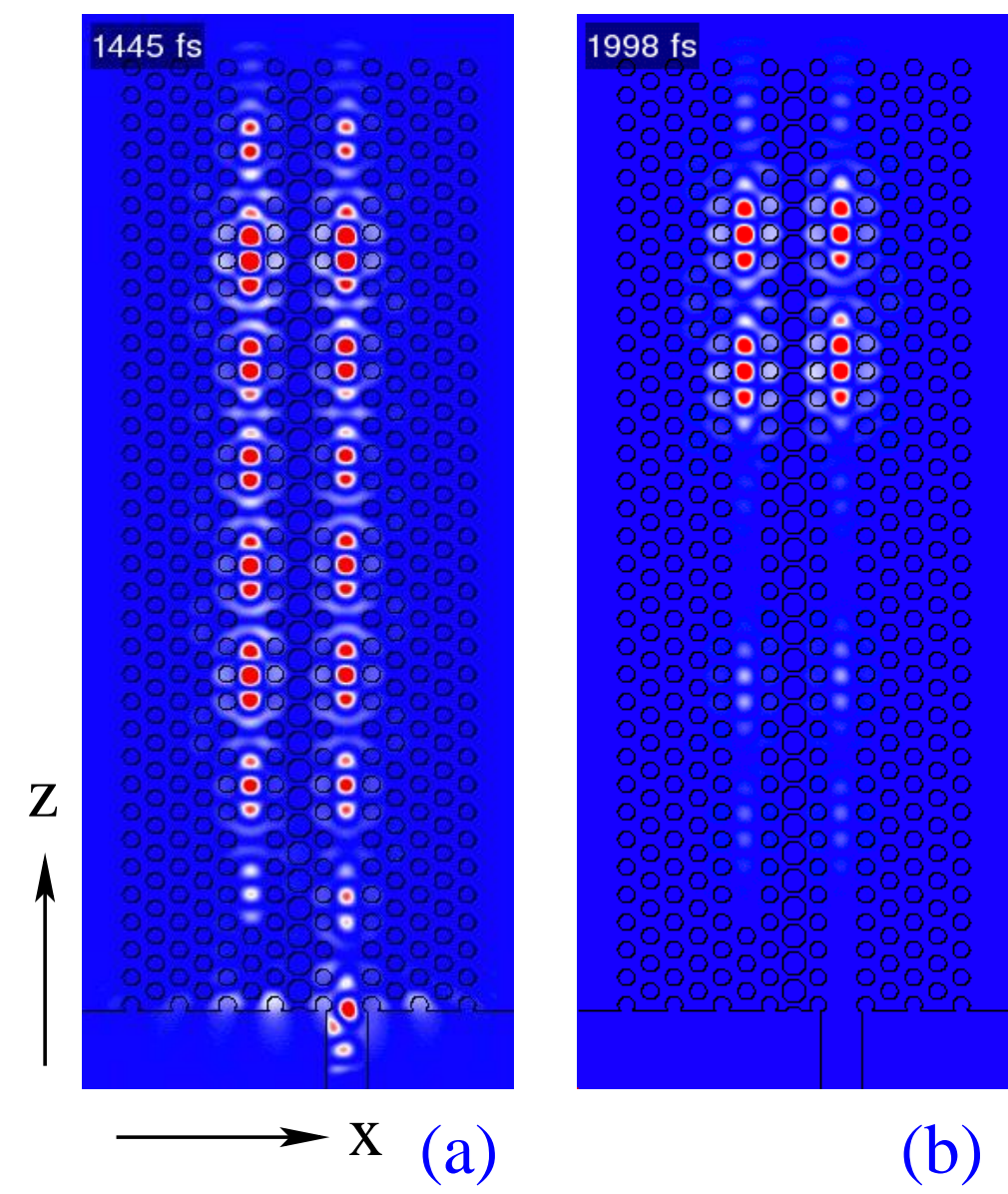

(b)

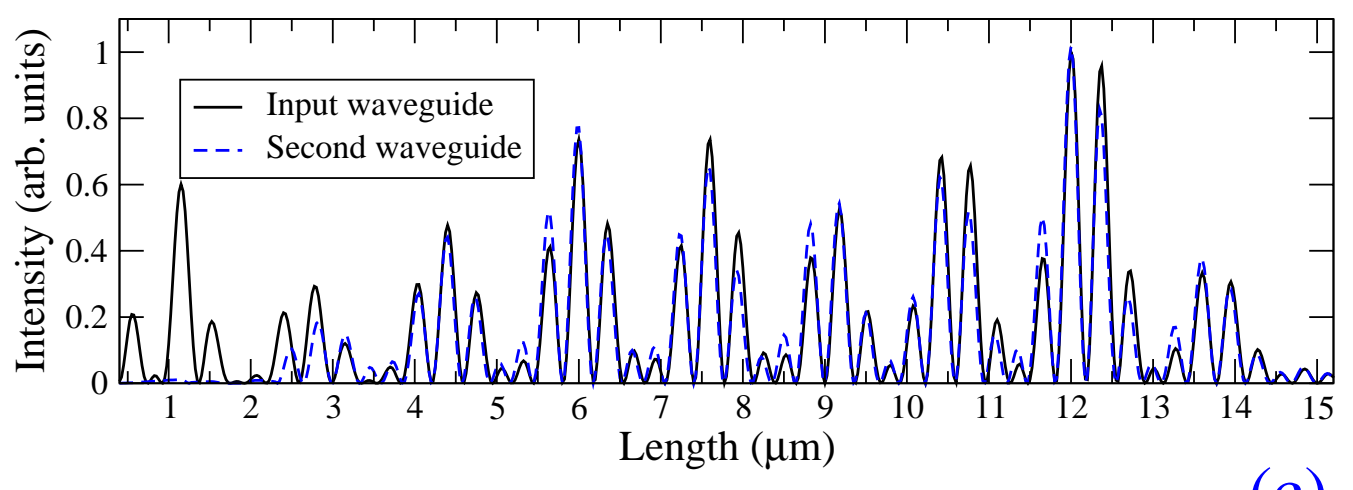

(c)

Figure 4. Simulations of pulse propagation through the symmetric coupler shown in Fig. 2(c), top. The central frequency of the pulse $(\omega=0.2197)$ is tuned close to the gap-edge, indicated by the open circle in 2(c), bottom. The estimated pulse velocity is $V_{g} \simeq c / 60$. Parameter values and the notation are as given in Fig. 3 . 


\section{Antisymmetric coupler ( $\mathrm{N}=2)$}

Intensity of mode 1

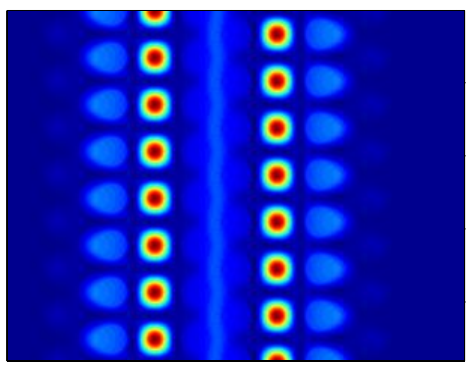

Phase of mode 1

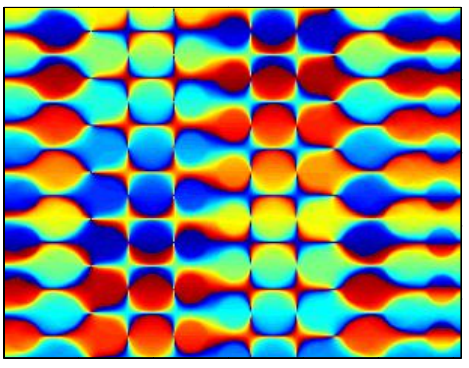

(a)
Intensity of mode 2

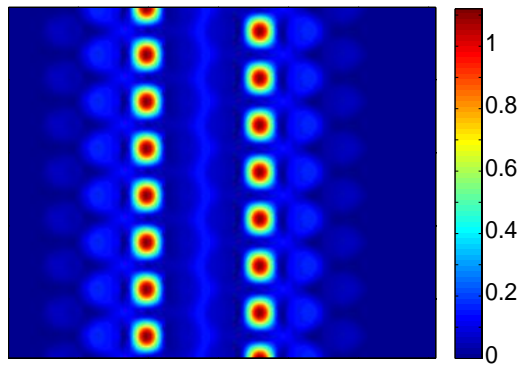

Phase of mode 2

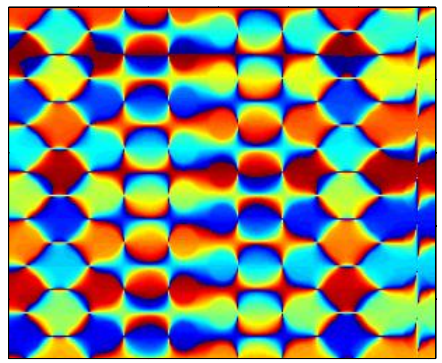

(b)
Intensity of mixed-mode excitation

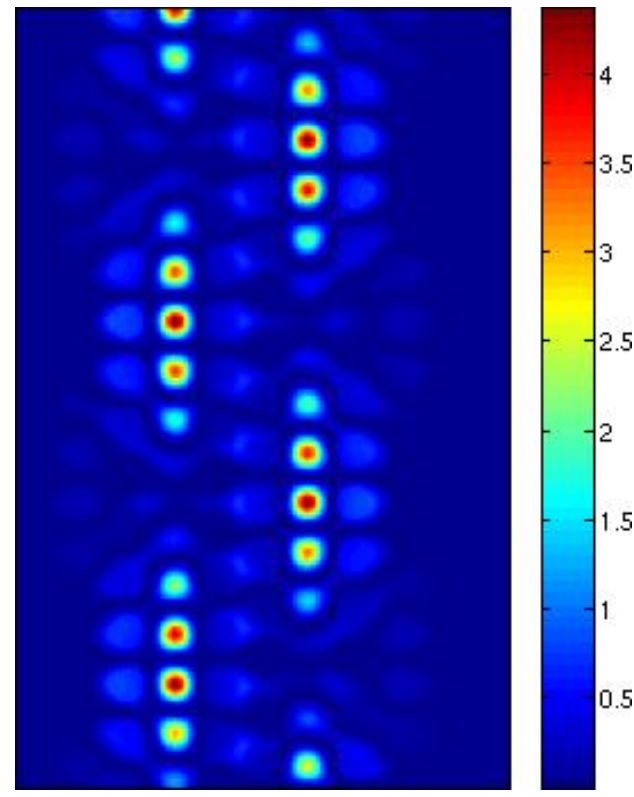

(c)

Figure 5. (a,b) Intensity (top) and phase (bottom) of the transverse magnetic field distributions for the band-edge modes of antisymmetric coupler [shown in Fig. 2(b)] at $\omega \simeq 0.214$ with (a) positive $(k=0.44)$ and (b) negative $(k=-0.41)$ wavenumbers, respectively. (c) Intensity of the simultaneously excited modes.

the lattice constant), as shown in Fig. 2(c). As a result, there now coexist two forward-propagating slow-light modes close to the band-edge. The FDTD simulations show that, indeed, there appears to be a periodic beating between the modes along the coupler, see Fig. 4. An interesting feature is that the positions of minima and maxima along the waveguides remain the same at all time steps as the pulse gradually propagates through the structure. This is a key signature of Bloch-wave interference, in contrast to the partial pulse splitting effect for the $N=1$ coupler where the field minima move along the waveguide together with the optical pulse. For the $N=3$ coupler, however, the oscillations are still synchronous in both waveguides and the energy is again equally distributed between the coupler arms because two co-propagating Bloch modes have the same symmetry, confirming the conclusions of Sec. 2.

\section{ANTISYMMETRIC COUPLER FOR SLOW-LIGHT}

Since the dispersion of the antisymmetric coupler shown in Fig. 2(b) has a band-edge inside the Brillouin zone, it remains for us to check condition (ii) for mode symmetry, formulated in Sec. 2. We have performed high-precision calculations in the regime of slow light, i.e. near the cut-off frequencies of defect modes, by using a Bloch mode scattering matrix approach. ${ }^{13,14}$

Characteristic mode profiles calculated close to the band-edge are shown in Figs. 5(a,b). We see that whereas the intensity patterns practically coincide, the phase structures have distinct symmetries. As a result, the beating of these modes realizes light switching between the waveguides, see Fig. 5(c). Such tunneling is facilitated solely by the coupler symmetry, without the need for any special structure optimization.

We now analyze the dependence of the coupling length and the group velocity on the frequency detuning from the band-edge. In agreement with the general analysis presented above in Sec. 2, the coupling length approaches a constant value as the detuning is decreased, and the group velocity varies by several orders of magnitude. At 


\section{Antisymmetric coupler $(\mathrm{N}=2)$}

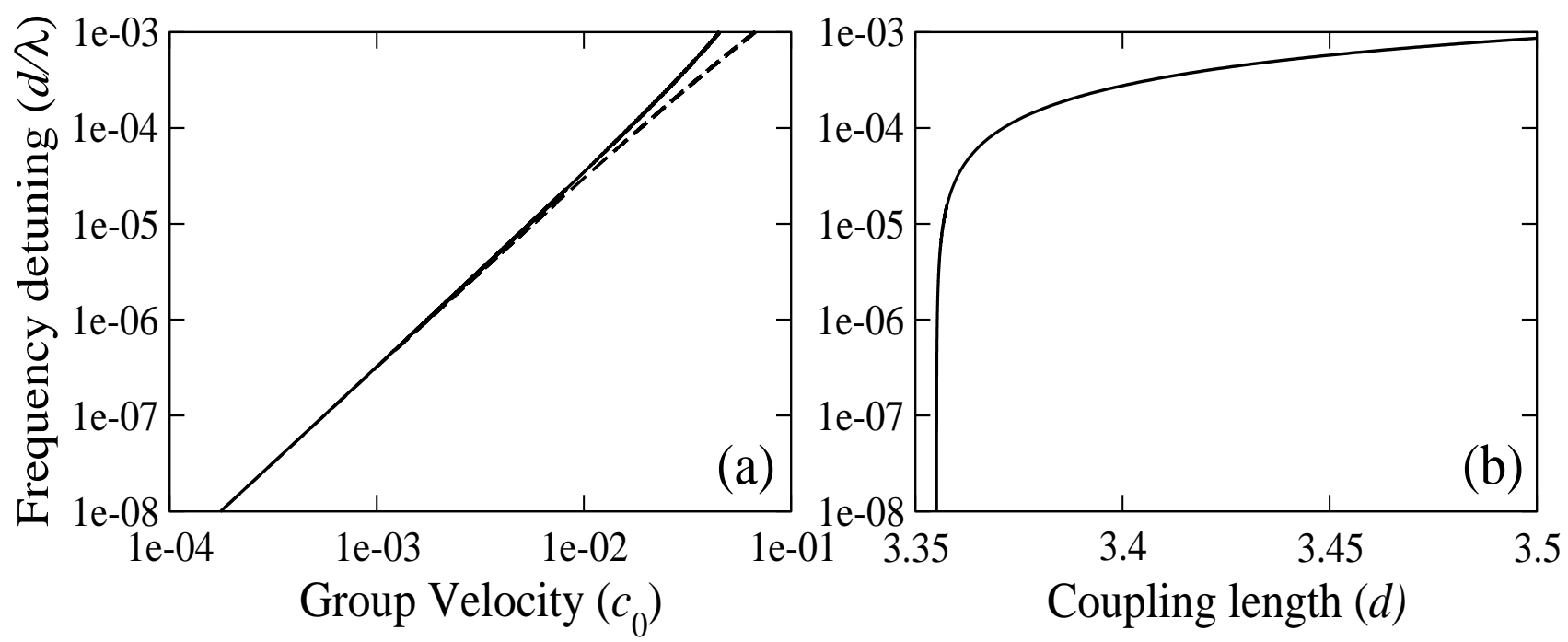

Figure 6. (a) Dependence of the group velocities on the frequency detuning from the band-edge (in logarithmic scale) shown for modes with positive (solid line) and negative (dashed line) wavenumbers, and (b) the corresponding dependence of the coupling length on frequency detuning.

the same time, the group velocities of the two modes become closely matched. This indicates that the coupler dynamics can also be observed for pulses tuned close to the band-edge, since the coupling length will be the same for all constituent spectral components.

We perform finite-difference time-domain (FDTD) simulations to investigate pulse propagation through the antisymmetric coupler, and confirm the predicted effect of dispersionless tunneling for slow-light pulses. The simulations at the band-edges may be especially sensitive to discretization methods, and we have additionally validated our results through the comparison of band-gap spectra with frequency-domain calculations. Examples presented in Fig. 7 show that the pulse periodically couples between the waveguides with the same coupling length for different detunings from the band-edge, and accordingly different propagation velocities. We determine the group velocity based on the distance traveled by the pulse as it propagates along the coupled waveguides, using the method introduced in Ref., ${ }^{3}$ and which generated good agreement with experimental measurements. In the first example shown in Figs. 7(a) and (b), the central wavelength is tuned close to the band-edge, and we find that $V_{g} \simeq c / 96$, where $c$ is the speed of light in vacuum. When the pulse spectrum is tuned slightly further away from the band-edge [Fig. 7 (c) and Fig. $7(\mathrm{~d})$ ], the group velocity is increased to $V_{g} \simeq c / 71$. According to the dependencies of field intensities along the centres of two coupled waveguides presented in Fig. 7(e), the switching contrast exceeds a factor of 50. Despite the dependence of the group velocity on the wavelength detuning, and the associated effect of dispersion, ${ }^{15}$ these results confirm that the slowlight pulse, as a whole, can be switched between the waveguides.

\section{CONCLUSIONS}

We have presented a novel and general approach to the design of photonic crystal couplers for slow light pulses. Extensive numerical simulations confirm that, in structures satisfying certain symmetry constraints, the pulses can be switched fully between parallel waveguides with coupling lengths of just a few unit cells, despite the effect of group velocity dispersion. We expect that our findings on slow light dynamics in antisymmetric photonic crystal couplers may enable further advances in active manipulation of slow light in integrated photonic structures. 


\section{Antisymmetric coupler $(\mathrm{N}=2)$}
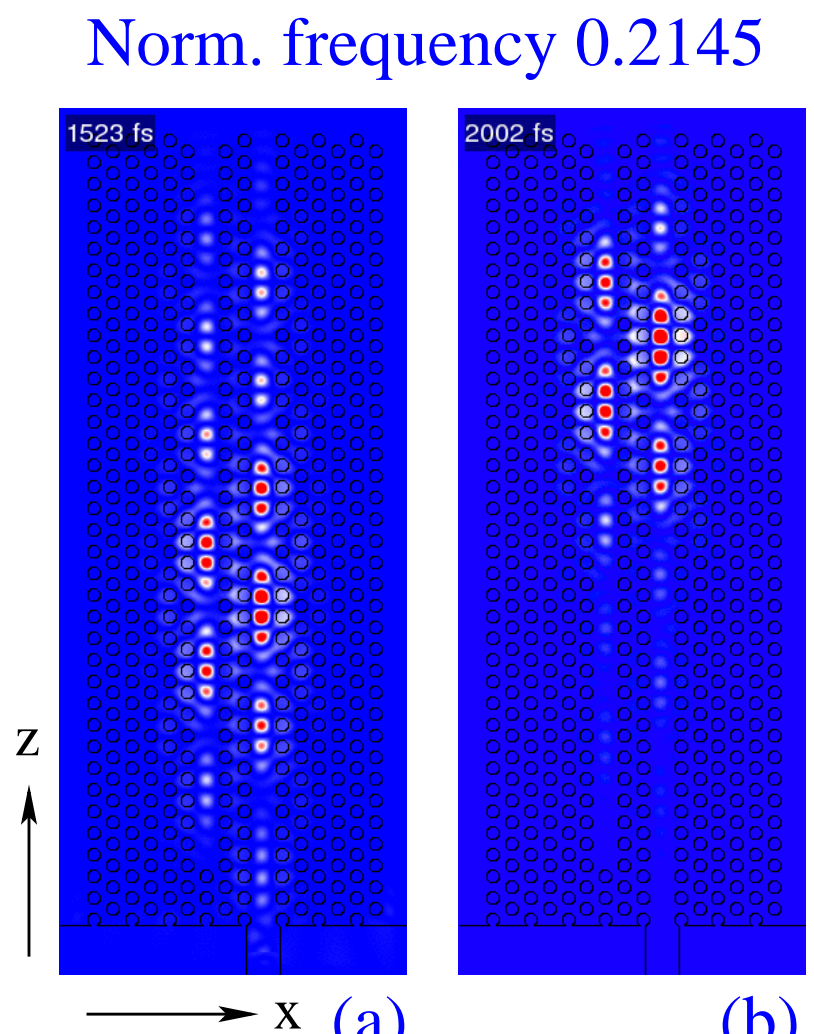

(a)

(b)

Norm. frequency 0.2156
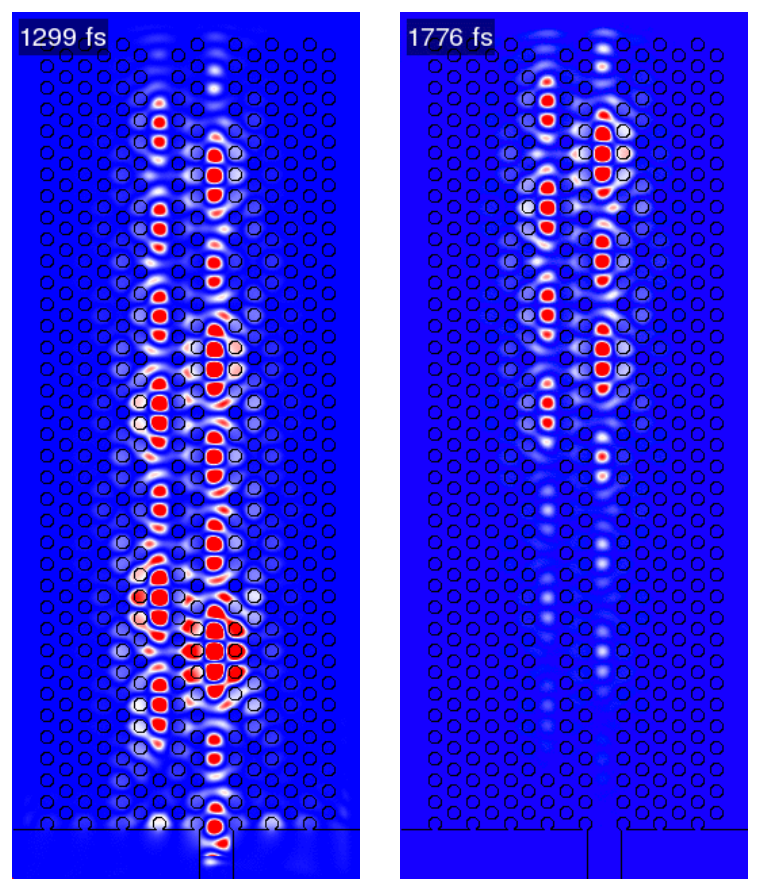

(c)

(d)

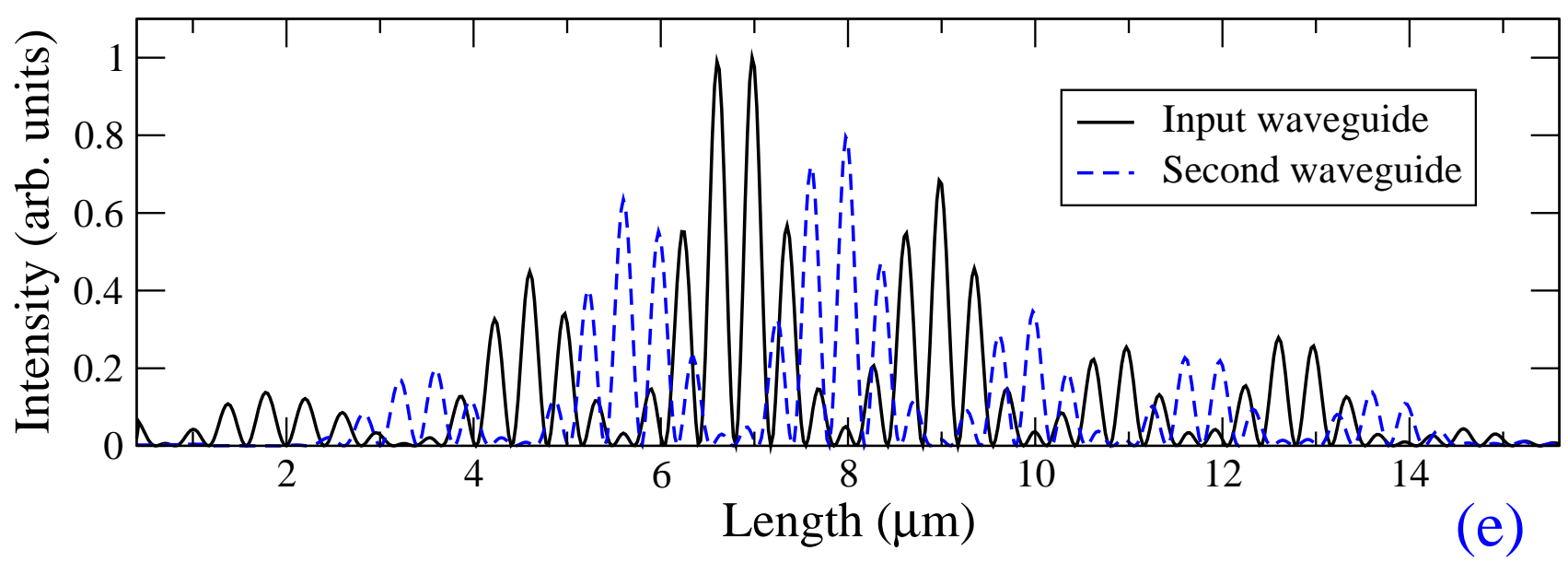

Figure 7. Simulations of pulse propagation through the antisymmetric coupler shown in Fig. 2(b), top. (a-d) Snapshots of the magnetic field intensity at different time-steps as indicated by the labels. (e) Field intensities along the centres of two coupled waveguides, corresponding to figure (a). The central wavelength of the pulse spectrum is (a,b,e) tuned close to the band-edge and (c,d) tuned further away from the band-edge as indicated by labels. The lattice constant is 400nm, the input pulse duration is 500fs, and the corresponding normalized pulse bandwidth $\Delta \omega=0.002$. The estimated pulse velocity is $(\mathrm{a}, \mathrm{b}, \mathrm{e}) V_{g} \simeq c / 96$ and $(\mathrm{c}, \mathrm{d}) V_{g} \simeq c / 71$. 


\section{ACKNOWLEDGMENTS}

This work was supported by the Australian Research Council under the Centres of Excellence Program, and through the Australian Partnership for Advanced Computing National Facility (APAC). D.N.C. acknowledges partial support by the Deutsche Forschungsgemeinschaft through projects SPP 1113 and FOR 557. A.V.L. acknowledges partial support from European Commission FP6, project NewTon, NMP4-CT-2005-017160.

\section{REFERENCES}

1. Y. A. Vlasov, M. O’Boyle, H. F. Hamann, and S. J. McNab, "Active control of slow light on a chip with photonic crystal waveguides," Nature 438, pp. 65-69, Nov 32005.

2. H. Gersen, T. J. Karle, R. J. P. Engelen, W. Bogaerts, J. P. Korterik, N. F. van Hulst, T. F. Krauss, and L. Kuipers, "Real-space observation of ultraslow light in photonic crystal waveguides," Phys. Rev. Lett. 94, pp. 073903-4, Feb 252005.

3. R. S. Jacobsen, A. V. Lavrinenko, L. H. Frandsen, C. Peucheret, B. Zsigri, G. Moulin, J. Fage-Pedersen, and P. I. Borel, "Direct experimental and numerical determination of extremely high group indices in photonic crystal waveguides," Opt. Express 13, pp. 7861-7871, Oct 32005.

4. M. D. Settle, R. J. P. Engelen, M. Salib, A. Michaeli, L. Kuipers, and T. F. Krauss, "Flatband slow light in photonic crystals featuring spatial pulse compression and terahertz bandwidth," Opt. Express 15, pp. 219-226, Jan 82007.

5. S. C. Huang, M. Kato, E. Kuramochi, C. P. Lee, and M. Notomi, "Time-domain and spectral-domain investigation of inflection-point slow-light modes in photonic crystal coupled waveguides," Opt. Express 15, pp. 3543-3549, Mar 192007.

6. A. V. Lavrinenko, A. Tetu, L. H. Frandsen, J. Fage-Pedersen, and P. I. Borel, "Optimization of photonic crystal 60 degrees waveguide bends for broadband and slow-light transmission," Appl. Phys. B 87, pp. 53-56, Mar 2007.

7. Y. Sugimoto, Y. Tanaka, N. Ikeda, T. Yang, H. Nakamura, K. Asakawa, K. Inoue, T. Maruyama, K. Miyashita, K. Ishida, and Y. Watanabe, "Design, fabrication, and characterization of coupling-strengthcontrolled directional coupler based on two-dimensional photonic-crystal slab waveguides," Appl. Phys. Lett. 83, pp. 3236-3238, Oct 202003.

8. D. Mori and T. Baba, "Dispersion-controlled optical group delay device by chirped photonic crystal waveguides," Appl. Phys. Lett. 85, pp. 1101-1103, Aug 162004.

9. D. Mori and T. Baba, "Wideband and low dispersion slow light by chirped photonic crystal coupled waveguide," Opt. Express 13, pp. 9398-9408, Nov 142005.

10. N. Yamamoto, T. Ogawa, and K. Komori, "Photonic crystal directional coupler switch with small switching length and wide bandwidth," Opt. Express 14, pp. 1223-1229, Feb 62006.

11. Y. J. Quan, P. D. Han, X. D. Lu, Z. C. Ye, and L. Wu, "Optical interleaver based on directional coupler in a 2D photonic crystal slab with triangular lattice of air holes," Opt. Commun. 270, pp. 203-206, Feb 152007.

12. S. G. Johnson and J. D. Joannopoulos, "Block-iterative frequency-domain methods for maxwell's equations in a planewave basis," Opt. Express 8, pp. 173-190, Jan 292001.

13. L. C. Botten, T. P. White, A. A. Asatryan, T. N. Langtry, C. M. de Sterke, and R. C. McPhedran, "Bloch mode scattering matrix methods for modeling extended photonic crystal structures. i. theory," Phys. Rev. E 70, pp. 056606-13, Nov 2004.

14. K. Dossou, M. A. Byrne, and L. C. Botten, "Finite element computation of grating scattering matrices and application to photonic crystal band calculations," J. Comput. Phys. 219, pp. 120-143, Nov 202006.

15. R. J. P. Engelen, Y. Sugimoto, Y. Watanabe, J. P. Korterik, N. Ikeda, N. F. van Hulst, K. Asakawa, and L. Kuipers, "The effect of higher-order dispersion on slow light propagation in photonic crystal waveguides," Opt. Express 14, pp. 1658-1672, Feb 202006. 